\title{
Characterization of the serum levels of Meteorin-like in patients with inflammatory bowel disease and its association with inflammatory cytokines
}

Afsane Gholamrezayi ${ }^{1 \dagger}$, Maryam Mohamadinarab ${ }^{2 \dagger}$, Pegah Rahbarinejad ${ }^{2}$, Soudabeh Fallah ${ }^{3}$, Shekufe Rezghi Barez ${ }^{4}$, Leila Setayesh ${ }^{5}$, Nariman Moradi ${ }^{6}$, Reza Fadaei ${ }^{7}$, Elham Chamani ${ }^{8^{*}}$ (D) and Tahmine Tavakoli ${ }^{9^{*}}$

\begin{abstract}
Background: Meteorin-like (Metrnl) is an adipokine with insulin sensitizing and anti-inflammatory properties that has been discovered recently. The relation among Metrnl, Inflammatory Bowel Disease (IBD), and obesity has been unexplored yet.

Methods: The present study was conducted on 54 healthy control, 42 Ulcerative Colitis (UC), and 43 Crohn's disease $(C D)$ patients who were diagnosed by pathological examination. In all participants, serum levels of adiponectin, Metrnl, interleukin (IL)-6, and Tumor necrosis factor (TNF-a) were measured using ELISA kits.

Results: Metrnl concentration was considerably lower in both UC ( $85.25 \pm 36.55 \mathrm{pg} / \mathrm{mL})$ and CD $(76.93 \pm 27.92 \mathrm{pg} /$ $\mathrm{mL})$ patients in comparison to control $(107.52 \pm 35.33 \mathrm{pg} / \mathrm{mL})$. In addition, it was seen that both patient groups have a decreased level of adiponectin compared to the controls. Besides that, the level of IL-6 and TNF-a were significantly greater in the patient groups. Moreover, the result showed that the level of Metrnl is inversely correlated with body mass index (BMI) in the controls and the patients. Metrnl levels are also inversely associated with IL-6, and TNF-a in both of the patient groups.
\end{abstract}

Conclusions: The current study is the first one reporting the decreased levels of Metrnl in serum among patients with IBD, which is inversely related with BMI, TNF-a, and IL-6. These results suggested a possible relation of Metrnl with the pathogenesis of IBD, particularly through inflammatory process, although further studies are warranted to dissect the possible mechanism.

Keywords: Meteorin-like, Inflammatory bowel disease, Ulcerative colitis, Crohn's disease, Interleukin, Tumor necrosis factor, Adiponectin

\footnotetext{
*Correspondence: Chamani.ec@gmail.com; t.tavakoli95238@gmail.com

${ }^{\dagger}$ Afsane Gholamrezayi and Maryam Mohamadinarab contributed equally to

this work.

${ }^{8}$ Cardiovascular Diseases Research Center, Department of Clinical

Biochemistry, Birjand University of Medical Sciences, Birjand, Iran

${ }^{9}$ Cardiovascular Diseases Research Center, Department of Internal Medicine,

Gastroenterology Section, Faculty of Medicine, Birjand University of Medical

Sciences, Birjand, Iran

Full list of author information is available at the end of the article
}

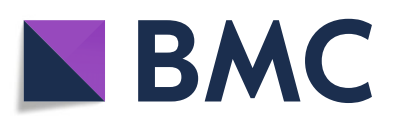

(- The Author(s). 2020 Open Access This article is licensed under a Creative Commons Attribution 4.0 International License, which permits use, sharing, adaptation, distribution and reproduction in any medium or format, as long as you give appropriate credit to the original author(s) and the source, provide a link to the Creative Commons licence, and indicate if changes were made. The images or other third party material in this article are included in the article's Creative Commons licence, unless indicated otherwise in a credit line to the material. If material is not included in the article's Creative Commons licence and your intended use is not permitted by statutory regulation or exceeds the permitted use, you will need to obtain permission directly from the copyright holder. To view a copy of this licence, visit http://creativecommons.org/licenses/by/4.0/. The Creative Commons Public Domain Dedication waiver (http://creativecommons.org/publicdomain/zero/1.0/) applies to the data made available in this article, unless otherwise stated in a credit line to the data. 


\section{Background}

Inflammatory Bowel Disease (IBD) as one of the causes of mortality in modern societies, characterized by gastrointestinal chronic inflammation $[1,2]$. Two most common and pathologic types of IBD are Crohn's disease and ulcerative colitis (UC). The exact etiology of IBD is not well-understood yet, therefore no-definitive cure has been identified [3]. In spite of previous research which showed that a possible association of underweight and malnutrition with the occurrence of IBD, recent studies have revealed about $15-40 \%$ of patients with IBD are obese and $20-40 \%$ are overweight which might contribute to the development of IBD [4]. Recent epidemiological studies have also shown that incidence of IBD is rising in parallel with obesity prevalence $[4,5]$. Moreover, the rate of hospitalization and surgery are more frequent among IBD patients with obesity [6]. Scientific studies have suggested that, genetics, gut microbiome, and the immune system may play critical roles in IBD, however, this has not completely understood yet [7]. IBD (UC and CD) also displays the characteristics of chronic inflammation and metabolic syndrome, that induces effective changes in metabolism $[8,9]$. Adipocytokines are secreted by white adipose tissue which have effect on the gut microbiome, inflammation, and metabolism pathways $[10,11]$. In a mutual way, IBD also can be considered as a risk factor for obesity via changing in the intestinal microbial metabolism $[9,12]$.

On the other hand, the impaired white adipose tissue (WAT) function such as abnormal adipocytokine secretion has a major and effective role on the inflammatory condition in colon tissue [12]. In this way, it was observed that, leptin concentration was decreased in the serum sample obtained from the IBD patients (with or without overweight). While the levels of resistin, adiponectin, and active ghrelin were remarkably increased [13].

Meteorin-like (Metrnl, known as Subfatin) is a novel adipo-myokine that is mainly expressed in WAT, however, it was reported that Metrnl is also expressed in colon epithelium. The anti-inflammatory function of Metrnl has been revealed recently and also has other roles including enhancing lipid metabolism, decreasing adipose inflammation, and ameliorating obesitymediated insulin resistance (IR) [14]. Notably, Metrnl expression was higher in mesenteric WAT of the CD patients in comparison to the controls [15]. Metrnl is highly expressed in intestinal cells, white adipose tissue, and skin and also expressed in other tissues including muscle, liver, heart, spleen, and central nervous system (CNS). Moreover, the activated macrophages produced Metrnl, which by this fact, it may be connected to the inflammatory disorders such as IBD [16]. A few studies have been conducted on tissue Metrnl levels in IBD disease. So far, no studies have been performed on investigating the association between serum Metrnl and IBD disease as well as the association among this protein, obesity, and the pro-inflammatory cytokines. Regarding to the fact that IBD is an inflammatory disease and Metrnl anti-inflammatory activities, in this study, the levels of Metrnl in serum were examined in the patients with inflammatory bowel disease and also its association was assessed with the hall-markers of inflammatory cytokines, interleukin (IL)-6, and Tumor necrosis factor (TNF- $\alpha$ ).

\section{Methods}

\section{Study population}

This case-control study was conducted on 54 control subjects (42 male and 12 female) and 85 IBD patients including 42 UC (31 male and 11 female) and 43 CD (28 male and 15 female) who were recruited from endoscopy unit of Valiasr Hospital, Birjand, Iran. All the individuals aged between 35 and 60 years old. The patient and control groups were selected by clinical examination, radiologic, endoscopic, and pathologic criteria. Normal people without any inflammatory diseases was considered as control group and based on age, gender and BMI were matched with patient groups. The inclusion criteria for patients were as following; were diagnosed by radiologic, endoscopic examination according to clinical and pathological guideline documents, and presence of other IBD's manifestations including diarrhea, abdominal pain, rectal bleeding, and malnutrition. In addition, none of them were received medication or anti-inflammatory drugs. All of patients were diagnosed recently. Written consent form was obtained from all participants. The diagnosis of UC/CD were based on established clinical and histopathological criteria. Moreover, the subjects were excluded with any history of cancer, diabetes, autoimmune diseases or active infectious disease.

\section{Anthropometric data and laboratory measurements}

Demographic data and medical history were obtained by a self-questionnaire from all participants. At the beginning of examinations, weight and height were taken from participants who were wearing light clothes, without shoes. Body mass index (BMI) was also calculated by body weight (kg) divided by height squared (m2), and studied targets were categorized into normal weight $(\mathrm{BMI}<25)$ and overweight $(\mathrm{BMI} \geq 25)$. Systolic and diastolic blood pressures of all the participants were measured using a standard sphygmomanometer after $15 \mathrm{~min}$ resting in a sitting posture. After $12-\mathrm{h}$ of fast, $5 \mathrm{~mL}$ of venous blood was obtained from all the participants and the serum was separated by centrifugation. Subsequently, fasting blood sugar (FBS) and lipid profiles including triglycerides (TG), total cholesterol (TC), Highdensity lipoprotein-cholesterol (HDL-C), and Low- 
density lipoprotein-cholesterol (LDL-C) were measured using auto-analyzer and the commercially available kits (Pars Azmoon, Tehran, Iran).

\section{Serum adipokine and cytokines}

Circulating levels of Metrnl were evaluated using an immunoassay kit (R\&D Systems, Minneapolis, USA, Cat\#DY7867). Moreover, the inter-assay and intra-assay variations were calculated as 6 and 8\%, respectively. Adiponectin serum levels were measured using an ELISA kit (Adipogen, Seoul, South Korea, Cat\#AG-45A0001YEK-K101) with inter- and intra-assay variations of 4.4 and $4.6 \%$, respectively. Afterward, the ultrasensitive ELISA kits were used to measure the serum levels of inteleukin-6 (IL-6) (R \& D Systems, Minneapolis, USA, Cat\# HS600B) and TNF- $\alpha$ (R \& D Systems, Minneapolis, USA, Cat\# DTA00C). Notably, the minimum detectable ranges of IL- 6 and TNF- $\alpha$ were obtained as 0.7 and 1.6 $\mathrm{pg} / \mathrm{mL}$. Inter and intra-assay variations of IL-6 were 9 and $7 \%$ and inter and also intra-assay variations of TNF$\alpha$ were 6 and 5\%, respectively.

\section{Statistical analysis}

Statistical analysis was performed using SPSS version 18 . Categorical data was analyzed using chi-square test and presented in frequency and percentage. Continuous variables were also examined by student $\mathrm{t}$-test and one-way ANOVA, and presented in mean and standard deviation (SD). Pearson correlation test was applied to correlation analysis. Furthermore, multinomial logistic regression was conducted to estimate the risk of diseases status according to serum levels of Metrnl.

\section{Results}

\section{Anthropometric and biochemical measurement}

The anthropometric and biochemical variables of the studied population are presented in Table 1. There is no significant difference in terms of age, sex, and BMI. In addition, there were no significant difference in the frequency of normal weight and overweight between the groups. Although FBS illustrated no significant difference between the controls and patients with UC and $\mathrm{CD}$, insulin and homeostatic model assessment for insulin resistance (HOMA-IR) were dramatically higher in the $\mathrm{CD}$ patients compared to the controls. It should be noted that, higher levels of insulin and HOMA-IR in the UC patients compared to the controls did not reach to the significant threshold. Furthermore, lipids profile including TG, TC, HDL-C, and LDL-C and systolic blood pressure (SBP) and diastolic blood pressure (DBP)

Table 1 Anthropometric and biochemical characteristic of studied population

\begin{tabular}{|c|c|c|c|c|}
\hline & Control $(n=54)$ & UC $(n=42)$ & $\mathrm{CD}(n=43)$ & $P$ value \\
\hline Sex (male/female) & $42 / 12$ & $31 / 11$ & $28 / 15$ & 0.373 \\
\hline BMI (kg/m2) & $24.12 \pm 3.56$ & $23.45 \pm 3.64$ & $24.43 \pm 4.61$ & 0.502 \\
\hline Normal weight / over weight & $29 / 25$ & $26 / 16$ & $21 / 22$ & 0.473 \\
\hline Age (year) & $39.02 \pm 4.6$ & $38.24 \pm 5.32$ & $38.74 \pm 5.7$ & 0.763 \\
\hline SBP $(m m H g)$ & $131.17 \pm 20.79$ & $133.40 \pm 24.26$ & $131.65 \pm 23.97$ & 0.887 \\
\hline $\mathrm{DBP}(\mathrm{mmHg})$ & $81.63 \pm 13.11$ & $83.10 \pm 14.48$ & $83.16 \pm 14.26$ & 0.825 \\
\hline $\mathrm{FBG}(\mathrm{mg} / \mathrm{dL})$ & $90.26 \pm 9.03$ & $93.65 \pm 13.27$ & $94.28 \pm 12.05$ & 0.172 \\
\hline Insulin ( $\mu \mathrm{U} / \mathrm{mL})$ & $4.03 \pm 0.31$ & $5.66 \pm 0.5$ & $5.93 \pm 0.64^{b^{*}}$ & 0.014 \\
\hline HOMA-IR & $0.89 \pm 0.07$ & $1.31 \pm 0.14$ & $1.43 \pm 0.17^{\mathrm{b}^{* *}}$ & 0.005 \\
\hline TG (mg/dL) & $117.41 \pm 44.83$ & $128.76 \pm 47.64$ & $126.08 \pm 38.15$ & 0.409 \\
\hline $\mathrm{TC}(\mathrm{mg} / \mathrm{dL})$ & $157.22 \pm 35.41$ & $164.54 \pm 47.49$ & $162.25 \pm 30.83$ & 0.629 \\
\hline LDL-C (mg/dL) & $94.70 \pm 26.95$ & $100.64 \pm 35.84$ & $104.93 \pm 21.74$ & 0.212 \\
\hline $\mathrm{HDL}-\mathrm{C}(\mathrm{mg} / \mathrm{dL})$ & $43.53 \pm 6.20$ & $42.11 \pm 9.07$ & $41.46 \pm 7.76$ & 0.394 \\
\hline Adiponectin $(\mu \mathrm{g} / \mathrm{mL})$ & $10.29 \pm 3.35$ & $7.88 \pm 2.83^{\mathrm{a}^{* *}}$ & $7.44 \pm 2.44^{\mathrm{b}^{* *}}$ & $<0.001$ \\
\hline TNF-a (pg/mL) & $16.08 \pm 5.50$ & $29.62 \pm 7.40^{\mathrm{a}^{* *}}$ & $30.43 \pm 8.25^{b^{* *}}$ & $<0.001$ \\
\hline IL-6 (pg/mL) & $6.38 \pm 3.51$ & $8.28 \pm 4.11$ & $9.17 \pm 4.18^{b^{* *}}$ & 0.002 \\
\hline Metrnl (pg/mL) & $107.52 \pm 35.33$ & $76.93 \pm 27.92^{\mathrm{a}^{* *}}$ & $85.25 \pm 36.55^{b^{* *}}$ & $<0.001$ \\
\hline
\end{tabular}

Data are presented as Mean \pm SD

One way ANOVA and Tukey's multiple comparisons test were used for comparison of quantitative variables

Abbreviations: UC ulcerative colitis, CD Crohn's disease, SBP systolic blood pressure, DBP diastolic blood pressure, FBG fasting blood glucose, HOMA-IR homeostasis model assessment of insulin resistance, $L D L-C$ low-density lipoprotein cholesterol, $H D L-C$ high-density lipoprotein cholesterol, $T C$ total cholesterol, $T G$ triglyceride

${ }^{\text {a }}$ Comparison between control and UC

${ }^{\mathrm{b}}$ Comparison between control and CD

${ }^{*} P<0.05$

${ }^{* *} P<0.01$ 

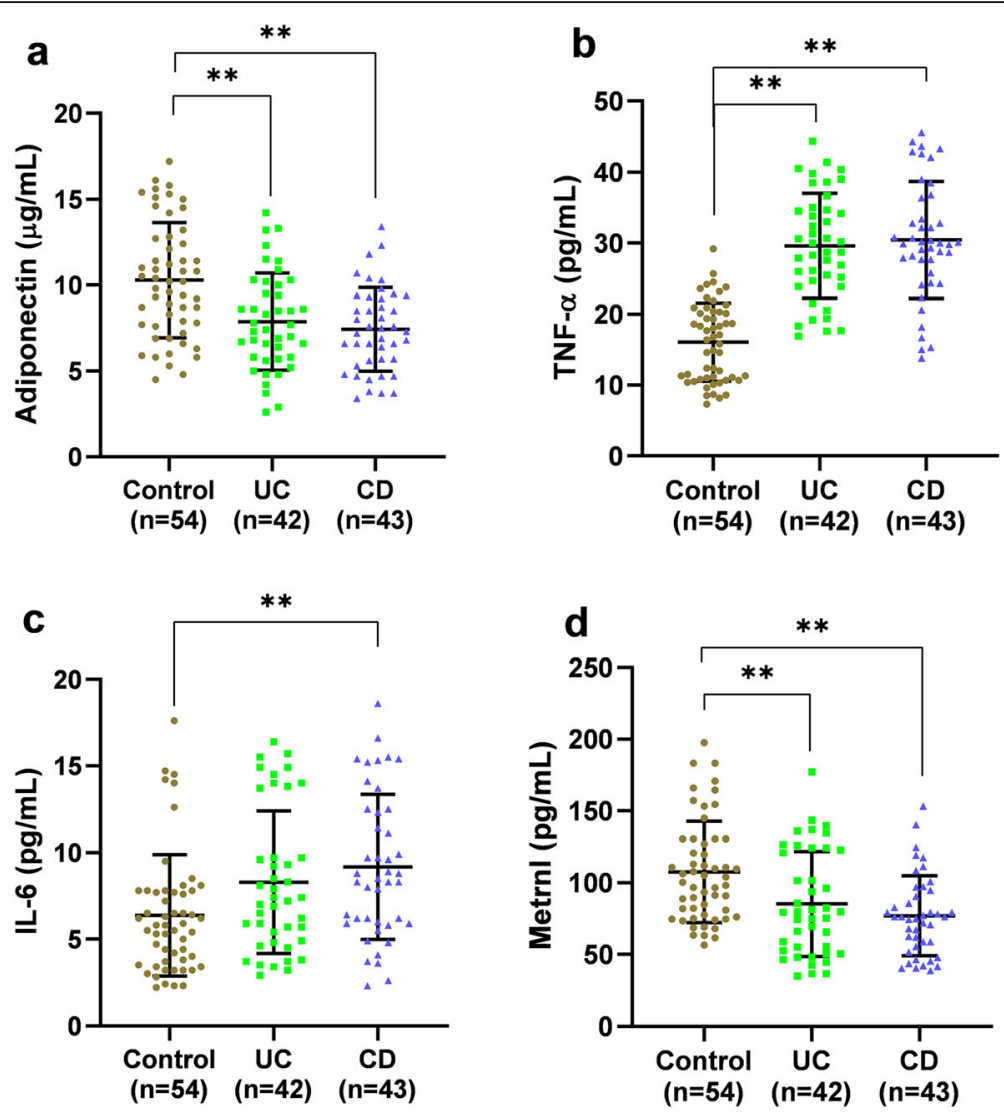

Fig. 1 Serum levels of adipokines and cytokines. a Serum levels of adiponectin decreased significantly in both UC and CD patients compared to controls. b Serum levels of TNF-alpha were found to be lower in both patient groups compared to controls. $\mathbf{c}$ IL-6 serum concentration indicated a considerable increase in CD group compared to controls. $\mathbf{d}$ Metrnl serum levels demonstrated a significant decline in UC and CD patients compared to controls. CD, Crohn's disease; IL-6, Interleukin 6; TNF-a, Tumor Necrosis Factor Alpha; UC, ulcerative colitis

demonstrated no considerable variation between the patients and controls.

\section{Serum levels of adipokines and cytokines}

The ELISA results (Fig. 1) showed that, adiponectin concentration was considerably lower in the patients with $\mathrm{UC}$ and $\mathrm{CD}$ compared to the controls. Furthermore, an elevated level of TNF- $\alpha$ in the patients in comparison to the controls was observed. Moreover, patients with $\mathrm{CD}$ showed higher IL-6 levels compared to the controls,

Table 2 Serum levels of Metrnl according to BMI cutoff

\begin{tabular}{llll}
\hline Group & Normal weight & Overweight & $\boldsymbol{P}$ \\
\hline All participants & $102.27 \pm 36.23$ & $78.13 \pm 31.04$ & $<0.001$ \\
Control & $117.84 \pm 37.53$ & $95.56 \pm 28.90$ & 0.019 \\
UC & $96.92 \pm 35.63$ & $66.29 \pm 30.25$ & 0.007 \\
CD & $87.40 \pm 27.24$ & $66.93 \pm 25.24$ & 0.014 \\
\hline
\end{tabular}

Data are presented as Mean \pm SD

ANOVA test was used to assess Metrnl levels, among three groups based on $\mathrm{BMI}$ cutoff (Overweight, $\mathrm{BMI} \geq 25$ and normal weight, $\mathrm{BMI}<25$ )

Abbreviations: UC ulcerative colitis, $C D$ Crohn's disease while this figure for the serum levels of IL-6 did not reach to the significant threshold in the patients with UC. Furthermore, a decreased concentration of Metrnl serum was observed in the patients with $\mathrm{UC}$ and $\mathrm{CD}$ compared to the controls.

Regarding the crucial role of adipose tissue on Metrnl levels, analysis was performed according to the BMI cutoff (Overweight, BMI $\geq 25$ and normal weight, $\mathrm{BMI}<$ 25). In this regard, Metrnl serum levels were found to be lower in all the overweight subgroups (Table 2).

\section{Association of serum Metrnl with the risk of diseases status}

Multinomial logistic regression was performed to assess the risk of diseases status according to serum levels of Metrnl. The results demonstrated a significant association between the decreased levels of Metrnl with the risk of UC and CD diseases. Furthermore, these associations have been adjusted for confounding factors including age, sex and BMI and the relationships remained as significant for both UC and CD diseases (Table 3). 
Table 3 Odd ratio of diseases status according to 10 unit change in the serum levels of Metrnl

\begin{tabular}{llllll}
\hline Model & Group & Odd Ratio $\mathbf{9 5 \%}$ Cl) & $\boldsymbol{\beta}$ (standard error) & $\boldsymbol{P}$ value & Correct prediction (\%) \\
\hline Crude & UC & $0.833(0.735-0.944)$ & $-0.183(0.064)$ & 0.004 & 57.1 \\
& CD & $0.760(0.661-0.874)$ & $-0.275(0.071)$ & $<0.001$ & 69.8 \\
Model 1 & UC & $0.794(0.692-0.912)$ & $-0.230(0.070)$ & 0.001 & 57.1 \\
& CD & $0.738(0.636-0.858)$ & $-0.303(0.076)$ & $<0.001$ & 58.1 \\
\hline
\end{tabular}

Abbreviations: UC ulcerative colitis, CD Crohn's disease, $C$ Confidence interval

Model 1. Adjusted for age, sex and BMI

\section{Correlation analysis}

Correlation analyses were performed in 2 subgroups, as controls and patients and the results are presented in Table 4. In the control group, Metrnl was found to be inversely correlated with BMI (Fig. 2). Metrnl also had an inverse correlation with BMI, IL-6, and TNF- $\alpha$, and a positive correlation with FBG in the patient groups (Fig. 2).

\section{Discussion}

Adipokines, have regulatory roles on expression and secretion of various cytokines, by this fact they have significant effects on the immune system. Therefore, they can play a crucial role in inflammatory diseases like IBD, which also have a metabolic background [17]. Several studies have shown that, adipokines such as leptin, resistin, visfatin, retinol-binding protein-4, adiponectin,

Table 4 Correlation analysis of serum Metrnl levels with anthropometric and biochemical variables

\begin{tabular}{lll}
\hline & Metrnl & \\
\cline { 2 - 3 } & Control & IBD \\
\hline BMl (kg/m2) & $-0.298^{*}$ & $-0.391^{* *}$ \\
Age (year) & 0.174 & -0.146 \\
SBP (mmHg) & -0.026 & 0.032 \\
DBP (mmHg) & -0.110 & -0.061 \\
FBG (mg/dl) & 0.023 & $0.222^{*}$ \\
Insulin (uU/ml) & -0.184 & 0.085 \\
HOMA-IR & -0.175 & 0.127 \\
TG (mg/dl) & 0.202 & -0.004 \\
TC (mg/dl) & 0.082 & -0.016 \\
LDL-C (mg/dl) & 0.026 & -0.061 \\
HDL-C (mg/dl) & 0.085 & -0.029 \\
Adiponectin (ug/ml) & -0.012 & -0.076 \\
TNF-a (pg/mL) & 0.137 & $-0.380^{* *}$ \\
IL-6 (pg/ml) & -0.027 & $-0.324^{* *}$ \\
\hline
\end{tabular}

Abbreviations: UC ulcerative colitis, CD Crohn's disease, SBP systolic blood pressure, DBP diastolic blood pressure, FBG fasting blood glucose, HOMA-IR homeostasis model assessment of insulin resistance, $L D L-C$ low-density lipoprotein cholesterol, HDL-C high-density lipoprotein cholesterol, TC total cholesterol, TG triglyceride

${ }^{*} P<0.05$

${ }^{* *} P<0.01$ glucose, and insulin are deregulated in the IBD patients [13]. Metrnl is a novel adipokine, which has a major role in improvement of inflammation and insulin resistance improvement [18]. Accordingly, this adipokine has been investigated in several metabolic and inflammatory diseases. Lee et al. showed that, patients with diabetes mellitus type 2 (T2DM) had lower level of Metrnl in their serum [19]. Similarly, Dadmanesh et al. found that Metrnl concentration in the serum of patients with coronary artery disease and T2DM were lower [20]. While, Chung et al. reported that levels of Metrnl were significantly higher in T2D patients [21]. Results of Wanng et al, also represented increase in serum level of Metrnl in T2M patients in comparison to control. They proposed increase in serum Metrnl could strengthen the risk of T2DM independent of insulin resistance [22]. This contradiction might be a results from the difference in study population, diabetes duration and medication. Most of the previous studies have focused on the tissues level of Metrnl in inflammatory disorders, thus there is no data on the serum levels of Metrnl in these complications. Bridgewood et al. investigated the level of Metrnl in synovial tissue in the patients with Rheumatoid Arthritis, Psoriatic Arthritis, and Osteoarthritis. As a result, they found the elevated level of Metrnl in Psoriatic Arthritis [23]. To the authors knowledge, so far, current study is the novel one that reporting the serum levels of Metrnl in the IBD patients. In addition, the results show the lower serum levels of Metrnl in the IBD patients compared to the controls. However, Metrnl was not different between patients groups. Li et al. demonstrated that, Metrnl is highly expressed in the gastrointestinal tract of normal donors as well as mice. On the other hand, they produced intestinal epithelial cell-specific knockout mice, in spite of reduction in Metrnl expression in the gastrointestinal tract, it's level is not decreased in serum [16]. A recent study performed by Zuo et al. reported that Metrnl expression is higher in mesenteric adipose tissue (MAT) of the CD patients compared to the controls. They also showed that, systemic treatment of Metrnl can improve the adipocyte function, and reduce the macrophage infiltration and inflammation by acting on the peroxisome proliferator-activated receptors (PPAR $\gamma$ ) pathway in mice. Therefore, they 
a

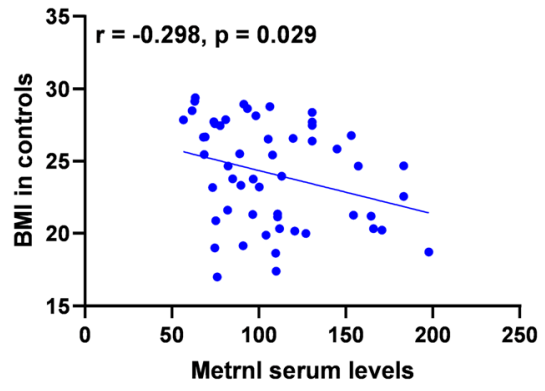

b

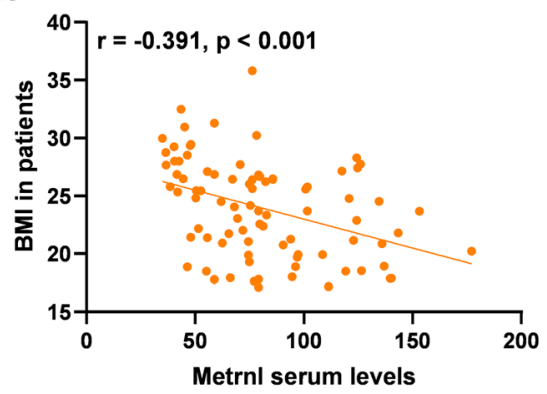

d

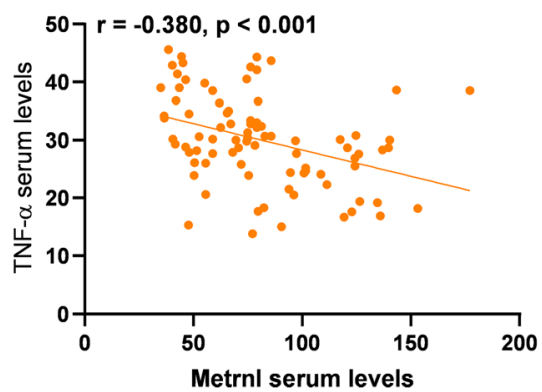

C

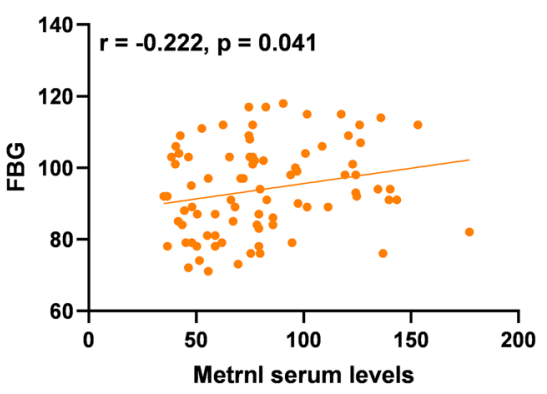

e

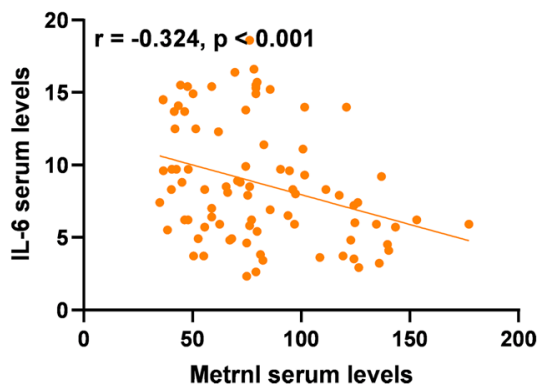

Fig. 2 The correlation of Metrnl with a) BMI in controls, b) BMI in patients, c) FBG in patients, d) TNF-a in patients and e) IL-6 in patients. BMI, body mass index; TNF-a, Tumor Necrosis Factor Alpha; IL-6, Interleukin 6

suggested that, upregulation of Metrnl in the MAT of the patients may be a compensatory response [14]. Regarding the inconsistent results, it seems likely that, Metrnl expression can have an organ dependent pattern; however, further longitudinal research is needed to support this hypothesis.

Furthermore, present study indicated an inverse relationship of Metrnl with the inflammatory cytokines in the IBD patients. It was also observed that, Metrnl may have regulatory action in inflammation pathways. Zuo et al. administered the Metrnl in IL-10-/- mice and then observed a significant decrease in the score of inflammation and pro-inflammatory factors such as TNF- $\alpha$, interferon (IFN)- $\gamma$, and IL-6 [12]. Additionally, Zhiyong LI et al. reported that, Metrnl plays a regulatory role in the expression of antimicrobial peptides such as islet-derived 3 gamma (Reg3g), lactotransferrin, and amyloid A-3 (SAA3) [23]. Since TNF- $\alpha$ and IL-6 are considered as the markers of inflammation, the results suggest a relationship between Metrnl and IBD pathogenesis. In addition, adiponectin decreased in the patient's groups, however, there were no relation between Metrnl and adiponectin that suggested a different regulation of these two adipokines.

When the population were stratified based on obesity, the serum level of Metrnl was significantly lower in 
obese subjects than in non-obese ones. Consistently, AlKhairi et al. reported that, Metrnl is significantly higher in the T2DM obese patients, in a way that this elevation can be explained as a compensatory response [24]. However, Zhi-Yong Li et al. showed no correlation between serum Metrnl levels and BMI [25]. As the adipose tissue is the main source of Metrnl secretion, it is expected that, BMI can affect the levels of this adipokine, and adipose tissue inflammation and dysfunction may be considered as the causes for the decrease in Metrnl levels.

\section{Study strength and limitations}

The present study for the first time measured Metrnl serum levels in patients $\mathrm{UC}$ and $\mathrm{CD}$ disease and its relation with inflammatory cytokines. The studied population were matched in terms of age, sex and BMI with control group that eliminated the impact of these confounding factor on the results. On the other hand, the present study has no data on body fat distribution that could be more clinically significant than BMI. Furthermore, the cross-sectional design of the study limited us in concluding a cause and effect relationship, so further studies are waranted to dissect the possible mechanism for the reported relationship.

\section{Conclusion}

In conclusion, the current study for the first time showed that a decreased level of Metrnl in the serum of IBD patients. Moreover, it was found that, serum level of Metrnl has a negative correlation with levels of TNF- $\alpha$, IL-6 and BMI in the serum of patients with IBD. Altogether, the present study found that Metrnl, inflammation, and obesity are related. This finding may suggest a possible association of Metrnl with the pathogenesis of IBD which can be considered for ameliorating the inflammatory milieu in these patients.

\section{Abbreviations \\ Metrnl: Meteorin-like; IBD: Inflammatory bowel disease; CD: Crohn's disease; IL-6: Interleukin 6; TNF-a: Tumor necrosis factor a; WAT: White adipose tissue; TG: Triglyceride; Chol: Cholesterol; FBS: Fasting blood sugars; SD: Standard deviations; T2DM: type 2 diabetes mellitus; HOMA-IR: Homeostatic model assessment- insulin resistance; SBP: Systolic blood pressure; DPB: Diastolic blood pressure}

\section{Acknowledgments}

The authors are thankful to research council of Birjand University of Medical Sciences for financial support.

\section{Authors' contributions}

Afsane Gholamrezayi \& Maryam Mohamadinarab: Design and performed experiments, analyzed data and co-wrote the paper. Pegah Rahbarinejad, Shekufe Rezghi Barez, Leila Setayesh: Performed experiments; Soudabeh Fallah: super vision and revised final manuscript and confirmed; Nariman Moradi and Reza Fadaei: Design experiment, Monitoring the experiment \&written the manuscript and revision; *Elham Chamani \& Tahmine Tavakili: Performed Endoscopy, collected samples, supervision, financial support \& corresponding Authors. All authors read and approved final manuscript.
Funding

The present study was supported by research council of Birjand University of Medical Sciences.

\section{Availability of data and materials \\ Additional data are available from the corresponding authors for reasonable requesting.}

\section{Ethics approval and consent to participate}

The written informed consent was signed by all the participants and the research was confirmed by the Ethics Committee of Birjand University of Medical Sciences.

\section{Consent for publication \\ No applicable.}

\section{Competing interests}

The authors declare no conflict of interest.

\section{Author details}

'Department of Nutrition, School of Public Health-International Campus, Iran University of Medical Sciences, Tehran, Iran. 'Department of Nutrition, Science and Research Branch, Islamic Azad University, Tehran, Iran. ${ }^{3}$ Department of Clinical Biochemistry, Faculty of Medicine, Iran University of Medical Sciences, Tehran, Iran. ${ }^{4}$ Department of Clinical Biochemistry, School of Pharmacy and Pharmaceutical Sciences, Isfahan University of Medical Sciences, Isfahan, Iran. ${ }^{5}$ Department of Community Nutrition, School of Nutritional Sciences and Dietetics, Tehran University of Medical Sciences (TUMS), Tehran, Iran. ${ }^{6}$ Cellular and Molecular Research Center, Research Institute for Health Development, Kurdistan University of Medical Sciences, Sanandaj, Iran. ${ }^{7}$ Sleep Disorders Research Center, Kermanshah University of Medical Sciences, Kermanshah, Iran. ${ }^{8}$ Cardiovascular Diseases Research Center, Department of Clinical Biochemistry, Birjand University of Medical Sciences, Birjand, Iran. ${ }^{9}$ Cardiovascular Diseases Research Center, Department of Internal Medicine, Gastroenterology Section, Faculty of Medicine, Birjand University of Medical Sciences, Birjand, Iran.

Received: 2 July 2020 Accepted: 19 October 2020

Published online: 30 October 2020

\section{References}

1. Kassam Z, Belga S, Roifman I, Hirota S, Jijon H, Kaplan GG, Ghosh S, Beck PL. Inflammatory bowel disease cause-specific mortality: a primer for clinicians. Inflamm Bowel Dis. 2014;20:2483-92.

2. Ananthakrishnan AN, McGinley EL, Binion DG. Excess hospitalisation burden associated with Clostridium difficile in patients with inflammatory bowel disease. Gut. 2008:57:205-10.

3. Cheifetz AS. Management of active Crohn disease. JAMA. 2013;309:2150-8.

4. Singh S, Dulai PS, Zarrinpar A, Ramamoorthy S, Sandborn WJ. Obesity in IBD: epidemiology, pathogenesis, disease course and treatment outcomes. Nat Rev Gastroenterol Hepatol. 2017;14:110-21.

5. Harper JW, Zisman TL. Interaction of obesity and inflammatory bowel disease. World J Gastroenterol. 2016;22:7868-81.

6. Szilagyi A. Relationship(s) between obesity and inflammatory bowel diseases: possible intertwined pathogenic mechanisms. Clin J Gastroenterol. 2020;13:139-52.

7. Zhou M, He J, Shen Y, Zhang C, Wang J, Chen Y. New Frontiers in genetics, gut microbiota, and immunity: a Rosetta stone for the pathogenesis of inflammatory bowel disease. Biomed Res Int. 2017;2017:8201672.

8. Zietek T, Rath E. Inflammation meets metabolic disease: gut feeling mediated by GLP-1. Front Immunol. 2016;7:154.

9. Yorulmaz E, Adali G, Yorulmaz H, Ulasoglu C, Tasan G, Tuncer I. Metabolic syndrome frequency in inflammatory bowel diseases. Saudi J Gastroenterol. 2011;17:376-82.

10. Fadaei R, Moradi N, Kazemi T, Chamani E, Azdaki N, Moezibady SA, Shahmohamadnejad S, Fallah S. Decreased serum levels of CTRP12/adipolin in patients with coronary artery disease in relation to inflammatory cytokines and insulin resistance. Cytokine. 2019;113:326-31.

11. Scheideler M, Herzig S, Georgiadi A. Endocrine and autocrine/paracrine modulators of brown adipose tissue mass and activity as novel therapeutic 
strategies against obesity and type 2 diabetes. Horm Mol Biol Clin Invest. 2017;31:1-12.

12. Bryant RV, Schultz CG, Ooi S, Goess C, Costello SP, Vincent AD, Schoeman SN, Lim A, Bartholomeusz FD, Travis SPL, Andrews JM. Obesity in inflammatory bowel disease: gains in adiposity despite high prevalence of Myopenia and osteopenia. Nutrients. 2018;10:1192.

13. Bilski J, Mazur-Bialy A, Wojcik D, Surmiak M, Magierowski M, Sliwowski Z, Pajdo R, Kwiecien S, Danielak A, Ptak-Belowska A, Brzozowski T. Role of obesity, mesenteric adipose tissue, and Adipokines in inflammatory bowel diseases. Biomolecules. 2019;9(12):780.

14. Zuo L, Ge S, Ge Y, Li J, Zhu B, Zhang Z, Jiang C, Li J, Wang S, Liu M, et al. The Adipokine Metrnl ameliorates chronic colitis in II-10-/- mice by attenuating mesenteric adipose tissue lesions during spontaneous colitis. J Crohn's Colitis. 2019;13:931-41.

15. Jung TW, Lee SH, Kim H-C, Bang JS, Abd El-Aty AM, Hacımüftüoğlu A, Shin $Y K$, Jeong JH. METRNL attenuates lipid-induced inflammation and insulin resistance via AMPK or PPARS-dependent pathways in skeletal muscle of mice. Exp Mol Med. 2018;50:122.

16. Li Z-Y, Fan M-B, Zhang S-L, Qu Y, Zheng S-L, Song J, Miao C-Y. Intestinal Metrnl released into the gut lumen acts as a local regulator for gut antimicrobial peptides. Acta Pharmacol Sin. 2016;37:1458-66.

17. Weidinger C, Ziegler JF, Letizia M, Schmidt F, Siegmund B. Adipokines and their role in intestinal inflammation. Front Immunol. 2018;9:1974.

18. Fadaei R, Dadmanesh M, Moradi N, Ahmadi R, Shokoohi Nahrkhalaji A Aghajani H, Ghorban K. Serum levels of subfatin in patients with type 2 diabetes mellitus and its association with vascular adhesion molecules. Arch Physiol Biochem. 2020;126:335-40.

19. Lee JH, Kang YE, Kim JM, Choung S, Joung KH, Kim HJ, Ku BJ. Serum Meteorin-like protein levels decreased in patients newly diagnosed with type 2 diabetes. Diabetes Res Clin Pract. 2018;135:7-10.

20. Dadmanesh M, Aghajani H, Fadaei R, Ghorban K. Lower serum levels of Meteorin-like/Subfatin in patients with coronary artery disease and type 2 diabetes mellitus are negatively associated with insulin resistance and inflammatory cytokines. PloS one. 2018;13:e0204180.

21. Chung HS, Hwang SY, Choi JH, Lee HJ, Kim NH, Yoo HJ, Seo J-A, Kim SG, Kim NH, Baik SH. Implications of circulating Meteorin-like (Metrnl) level in human subjects with type 2 diabetes. Diabetes Res Clin Pract. 2018;136:1007.

22. Wang K, Li F, Wang C, Deng Y, Cao Z, Cui Y, Xu K, Lin P, Sun Y. Serum levels of meteorin-like (Metrnl) are increased in patients with newly diagnosed type 2 diabetes mellitus and are associated with insulin resistance. Med Sci Monit. 2019;25:2337-43.

23. Bridgewood C, Russell T, Weedon H, Baboolal T, Watad A, Sharif K, Cuthbert R, Wittmann M, Wechalekar M, McGonagle D. The novel cytokine Metrn//IL41 is elevated in psoriatic arthritis synovium and inducible from both entheseal and synovial fibroblasts. Clin Immunol. 2019;208:108253.

24. AlKhairi I, Cherian P, Abu-Farha M, Madhoun AA, Nizam R, Melhem M, Jamal M, Al-Sabah S, Ali H, Tuomilehto J, et al. Increased expression of Meteorinlike hormone in type 2 diabetes and obesity and its association with Irisin. Cells. 2019;8:1283.

25. Li ZY, Song J, Zheng SL, Fan MB, Guan YF, Qu Y, X J, Wang P, Miao CY. Adipocyte Metrnl antagonizes insulin resistance through PPARY signaling. Diabetes. 2015;64:4011-22.

\section{Publisher's Note}

Springer Nature remains neutral with regard to jurisdictional claims in published maps and institutional affiliations.

Ready to submit your research? Choose BMC and benefit from:
- fast, convenient online submission
- thorough peer review by experienced researchers in your field
- rapid publication on acceptance
- support for research data, including large and complex data types
- gold Open Access which fosters wider collaboration and increased citations
- maximum visibility for your research: over 100M website views per year
At BMC, research is always in progress.
Learn more biomedcentral.com/submissions

